\title{
Condicionamento fisiológico de sementes de couve-flor
}

\author{
Ana Lúcia P Kikuti; Julio Marcos Filho \\ USP-ESALQ, Dep ${ }^{\text {to }}$ Produção Vegetal, C. Postal 09, 13418-900 Piracicaba-SP; alkikuti@esalq.usp.br; jmarcos@esalq.usp.br
}

\section{RESUMO}

Esta pesquisa teve como objetivos definir o procedimento para o condicionamento fisiológico de sementes de couve-flor e verificar a influência do potencial fisiológico das sementes sobre os resultados desse tratamento. Seis lotes de sementes, três da cultivar Sharon e três da cultivar Teresópolis Gigante, foram submetidos ao hidrocondicionamento entre camadas de duas, quatro e seis folhas de papel toalha e ao osmocondicionamento entre camadas de quatro folhas de papel toalha umedecidas com solução de polietilenoglicol 6000 (potenciais de -0,1 e -0,2 MPa). Em todos os procedimentos os teores de água foram ajustados para 32 e $38 \%$, para a cultivar Sharon, e 34 e $41 \%$ para a cultivar Teresópolis Gigante. Os efeitos dos tratamentos sobre a germinação e emergência das plântulas foram comparados à testemunha (sem condicionamento). O hidrocondicionamento entre quatro e seis folhas, até a obtenção dos teores de água mais elevados, beneficiou a velocidade de germinação (VG) e a de emergência de plântulas (VE), nas duas cultivares. Na cultivar Sharon a VG foi de 14,6 (testemunha) em comparação a 16,4 nas sementes hidrocondicionadas, para os lotes de maior e menor potencial fisiológico; paralelamente, os valores da VE aumentaram de 8,9 para 11,2 e de 7,8 para 11,6, nos lotes de menor potencial fisiológico. Para a cultivar Teresópolis Gigante, a VG e a VE foram favorecidas pelo hidrocondicionamento, independentemente do potencial fisiológico dos lotes (a VG aumentou de 16,4 para 21,4 e, a VE, de 9,5 para 13,5). Conclui-se que o hidrocondicionamento entre quatro e seis folhas de papel toalha, até a obtenção de teores de água próximos aos da emissão da raiz primária é favorável à velocidade de germinação e de emergência de plântulas em couve-flor e que o potencial fisológico inicial dos lotes pode influenciar a resposta ao condicionamento fisiológico, dependendo da cultivar ou histórico dos lotes.

Palavras-chave: Brassica oleracea var. botrytis, hidrocondicionamento, osmocondicionamento, germinação, vigor.

\section{ABSTRACT}

\section{Priming seed treatment of cauliflower seeds}

This research was conducted to define an adequate procedure for cauliflower seed priming and to verify whether the seed physiological potential affects priming results. Six seed lots, three of the cultivar Sharon and three of Teresópolis Gigante, were submitted to hydropriming between two, four and six sheets of towel paper and to osmopriming between four sheets of towel paper moistened with polietilenoglycol 6000 solutions (-0,1 and -0,2 MPa potentials). In all priming procedures, the seed water content was adjusted to $32 \%$ or $38 \%$ for cv. Sharon and $34 \%$ or $41 \%$, for cv. Teresópolis Gigante seeds. The effects of treatments on germination and seedling emergence were compared to unprimed control. Results showed that hydropriming between four and six sheets of towel paper was efficient to favor speed of germination (VG) and speed of seedling emergence (VE) in both cultivars when seeds attained $38 \%$ (cultivar Sharon) and $41 \%$ (cultivar Teresópolis Gigante). VG increased from 14,6 (control) to 16,4 (hydroprimed seeds) in cv. Sharon seed lots of higher and lower physiological potential; the same for VE that incresead from 8,9 to 11,3 and from 7,8 to 11,6 in seed lots of lower physiological potential. In seeds of cv. Teresópolis Gigante, hydropriming promoted increases in the VG and VE, independently of seed physiological potencial; VG values ranged from 16,4 to 21,4 and the VE from 9,5 to 13,5 . Results showed that hydropriming between four and six sheets of towel paper, in which seeds attained moisture content close to the required for primary root protrusion is efficient to favor the speed of germination and seedling emergence of cauliflower seeds; the influence of vigor level on seed priming response is closely related to cultivar and seed lot management.

Keywords: Brassica oleracea var. botrytis, hydropriming, osmopriming, germination, vigor.

\section{(Recebido para publicação em 3 de abril de 2008; aceito em 8 de maio de 2009) (Received in April 3, 2008; accepted in May 8, 2009)}

\begin{abstract}
A técnica do condicionamento fisiológico foi desenvolvida visando principalmente à redução do período de germinação e de emergência das plântulas e à obtenção de estandes uniformes, especialmente quando as sementes são expostas a condições ambientais adversas. Esta técnica compreende a hidratação controlada das sementes até atingir nível suficiente para ativar os processos preparatórios essenciais à germinação, sem permitir a protrusão da raiz primária. Desse modo, a hidratação deve ocorrer em velocidade que permita a reparação e/ou reorganização das membranas, reduzindo riscos de danos relacio-
\end{abstract}

nados à rápida absorção de água (Santos \& Menezes, 2000).

A emissão da raiz primária sinaliza o início da fase III da curva de embebição e caracteriza a perda da tolerância das sementes de várias espécies à dessecação. Portanto, é necessário determinar o momento apropriado para finalizar o fornecimento de água para as sementes; se a interrupção no fornecimento de água à semente for prematura, a ativação do metabolismo pode ser insuficiente para uniformizar o desempenho da amostra, quando tardia, pode intensificar a possibilidade de reversão dos efeitos do condicionamento (Mar- cos Filho, 2005).

Os tratamentos condicionadores incluem a hidratação em soluções osmóticas, empregando substâncias como polietilenoglicol (PEG) ou sais, o condicionamento em matriz sólida, em que as sementes são colocadas em contato com material sólido (de baixo potencial mátrico) umedecido e a hidratação em água (hidrocondicionamento) ou em atmosfera úmida ou por meio de tambor rotativo.

Para sementes de couve-flor, Fujikura et al. (1993) relataram que tanto o hidrocondicionamento quanto $\mathrm{o}$ 
osmocondicionamento favoreceram a velocidade de germinação de sementes não envelhecidas. Porém, o hidrocondicionamento apresentou a vantagem de ser mais simples, barato e não envolver reagentes ou equipamentos sofisticados. Do mesmo modo, a hidratação (imersão direta em água) com aeração complementar, a $20^{\circ} \mathrm{C}$, durante 32 horas, seguida de secagem, resultou em melhor desempenho de sementes de couve-flor e couve-de-Bruxelas, envelhecidas e não envelhecidas (Thornton \& Powell, 1995). No entanto, esses autores não indicaram os teores de água que as sementes deveriam alcançar durante o condicionamento.

Dentre os fatores envolvidos na eficiência do condicionamento está o potencial fisiológico inicial da semente. $\mathrm{Na}$ literatura são encontrados resultados contraditórios quanto aos efeitos em lotes de couve-flor de elevado potencial fisiológico, sendo positivo para lotes de menor potencial fisiológico (Thornton \& Powell, 1995; Powell et al., 2000).

Apesar da existência de poucos trabalhos referentes ao condicionamento fisiológico de sementes de couve-flor, estes são de considerável importância. Falhas na emergência ou a formação de plântulas fracas podem causar sérios prejuízos ou acréscimos nos custos de produção. Tendo em vista a importância econômica dessa cultura e os possíveis benefícios do condicionamento fisiológico, esta pesquisa teve como objetivos definir o procedimento mais adequado para realizar o condicionamento fisiológico de sementes de couve-flor e verificar a influência do potencial fisiológico inicial das sementes sobre a resposta ao condicionamento.

\section{MATERIAL E MÉTODOS}

A pesquisa foi conduzida em laboratório da ESALQ, em Piracicaba-SP, utilizando-se sementes de couve-flor das cultivares Sharon e Teresópolis Gigante, cada uma representada por três lotes. Inicialmente, as sementes, provenientes da empresa Sakata Seed Sudamerica Ltda., produzidas no ano agrícola 2002, foram tratadas com Iprodione (Rovral) + Thiram, na propor- ção de $0,8 \mathrm{~g}$ de Rovral + 2 mL de Thiram $\mathrm{kg}^{-1}$ de semente. Em seguida, determinou-se o teor de água das sementes e avaliou-se o potencial fisiológico dos seis lotes, através dos testes: Determinação do teor de água $=$ realizada pelo método da estufa, a $105^{\circ} \mathrm{C}$ durante 24 horas, de acordo com as Regras para Análise de Sementes (Brasil, 1992). Os resultados foram expressos em porcentagem média para cada lote (base úmida); Germinação= Utilizaram-se quatro repetições de 50 sementes para cada lote, colocadas para germinar sobre duas folhas de papel mata-borrão (tipo Germibox), umedecidas com quantidade de água equivalente a 2,5 vezes a massa do substrato, no interior de caixas de plástico (11,0 x 11,0 x 3,5 cm), a 20-30 ${ }^{\circ} \mathrm{C}$. Foi adotado fotoperíodo diário de oito horas, coincidindo com a temperatura mais alta. As avaliações foram efetuadas de acordo com os critérios estabelecidos em Regras para Análise de Sementes (Brasil, 1992), contando e retirando diariamente do substrato as plântulas normais de cada repetição. Os resultados foram expressos em porcentagem média de plântulas normais para cada lote, dez dias após a semeadura. Calculou-se também a velocidade de germinação (Maguire, 1962); Emergência de plântulas $=$ foram utilizadas quatro repetições de 50 sementes, distribuídas em células individuais de bandejas de poliestireno ("isopor"), contendo substrato comercial. As bandejas foram mantidas em ambiente protegido, com irrigação a cada dois dias. Registrou-se o número de plântulas normais 14 dias após a semeadura, sendo os resultados expressos em porcentagem de plântulas emersas para cada lote; Envelhecimento acelerado (solução salina $)=$ conduzido conforme metodologia descrita por Marcos Filho (1999), porém adicionando-se $40 \mathrm{~mL}$ de solução saturada de cloreto de sódio $(\mathrm{NaCl})$ no interior de cada caixa de plástico (11,0 x 11,0 x 3,5 $\mathrm{cm}$ ), com o objetivo de proporcionar ambiente com umidade relativa do ar de $76 \%$. As caixas de plástico com as amostras de sementes pré-pesadas $(4,0 \mathrm{~g})$ foram mantidas em câmara do tipo "jaquetada" (modelo 3015 VWR Scientific) durante 48 h, a $45^{\circ} \mathrm{C}$. Após o período de envelhecimento, quatro repetições de 50 sementes de cada amos- tra foram submetidas ao teste de germinação (Brasil, 1992). A avaliação foi realizada cinco dias após a semeadura, calculando-se a porcentagem de plântulas normais e; Condutividade elétrica $=$ conduzido pelo método massal, utilizandose quatro subamostras de 25 sementes para cada lote. As amostras foram pesadas (precisão de $0,0001 \mathrm{~g}$ ), colocadas em copos plásticos contendo $25 \mathrm{~mL}$ de água destilada e mantidas em germinador durante seis horas, a $25^{\circ} \mathrm{C}$. As leituras da condutividade elétrica foram realizadas em condutivímetro DIGIMED DM-31 e os valores médios, para cada lote, expressos em $\mu \mathrm{S} \mathrm{cm}^{-1} \mathrm{~g}^{-1}$ de semente.

Após a determinação do potencial fisiológico inicial dos lotes, foi determinada a marcha de absorção de água pelas sementes com quatro repetições de 6,0 g de sementes para cada lote. As sementes de cada repetição foram distribuídas entre duas camadas de duas folhas de papel toalha, umedecidas com água em quantidade correspondente a 2,5 vezes o peso do papel seco. Essas folhas umedecidas foram colocadas sobre telas em caixas de plástico transparente $(11,0 \times 11,0 \times 3,5 \mathrm{~cm})$ contendo 40 mL de água, para manutenção da atmosfera úmida. Em seguida, foram colocadas em germinador a $20^{\circ} \mathrm{C}$, sendo realizadas pesagens em intervalos de 3 horas, até que ocorresse a emissão da raiz primária. A taxa de embebição foi calculada com base no peso inicial das sementes, conforme fórmula a seguir, descrita por Hampton \& TeKrony (1995):

$$
\text { P2: [(100-A)/(100-B)] x P1 }
$$

Onde $\mathrm{A}=$ teor de água inicial da semente (base úmida); $\mathrm{B}=$ teor de água desejado; P1= peso inicial das sementes $(\mathrm{g}) ; \mathrm{P} 2=$ peso final das sementes $(\mathrm{g})$.

Em seguida, as sementes foram submetidas ao condicionamento fisiológico. No hidrocondicionamento, as sementes foram embebidas entre duas camadas de uma, duas e três folhas de papel toalha, umedecidas com quantidade de água equivalente a 2,5 vezes a sua massa. Neste procedimento, as sementes foram mantidas a $20^{\circ} \mathrm{C}$, até atingirem teores de água de 32 e $38 \%$, para a cultivar Sharon, e 34 e $41 \%$, para a cultivar Teresópolis Gigante. Os teores de água 
foram determinados com base na marcha de absorção de água pelas sementes; os valores mais baixos corresponderam ao início e, os mais altos, ao fim da fase II de embebição. Atingidos os teores de água desejados, as sementes foram retiradas do substrato e submetidas à secagem superficial (sobre papel toalha) por 15 minutos, para posterior instalação dos testes de germinação e emergência das plântulas.

Para o osmocondicionamento, foram utilizadas soluções de polietilenoglicol 6000 (PEG 6000) com potenciais osmóticos ajustados para -0,1 $\mathrm{MPa}$ e -0,2 MPa (Villela et al., 1991). Os potenciais osmóticos foram selecionados de modo a permitirem que as sementes atingissem os teores de água esperados (32 e 38\%, para a cultivar Sharon, e 34 e $41 \%$, para a cultivar Teresópolis Gigante). Para cada potencial osmótico, as sementes foram colocadas entre duas camadas de duas folhas de papel toalha, umedecidas com quantidade de solução equivalente a 2,5 vezes a massa do papel, a $20^{\circ} \mathrm{C}$. As sementes permaneceram no germinador até atingirem os teores de água esperados, sendo em seguida, retiradas do substrato, lavadas em água corrente e submetidas à secagem superficial (sobre papel toalha), por 15 minutos, para posterior instalação dos testes de germinação e de emergência de plântulas.

Para a avaliação dos efeitos imediatos do condicionamento foram utilizados os testes de germinação e de emergência de plântulas (velocidade e porcentagem), conforme descrito anteriormente, com a diferença de que, no teste de emergência, a avaliação foi realizada diariamente, através da contagem de plântulas normais em que o comprimento da parte aérea era igual ou superior a $1,0 \mathrm{~cm}$. Os resultados foram expressos em valores médios de velocidade (Maguire, 1962) e em porcentagem de emergência de plântulas 14 dias após a semeadura.

A análise estatística dos dados foi realizada considerando-se separadamente cada cultivar e teste conduzido. Utilizou-se delineamento inteiramente casualizado, com quatro repetições e tratamentos dispostos em fatorial $10 \times 3$ (dez tratamentos e três lotes) para a cultivar Sharon ou $9 \times 3$ (nove tratamentos

Tabela 1. Testes de germinação, velocidade de germinação, emergência de plântulas, envelhecimento acelerado, condutividade elétrica e teor de água de seis lotes de sementes de couve-flor, cultivares Sharon e Teresópolis Gigante (germination test, speed of germination, seedling emergence, aging accelerated, electrical conductivity and moisture content of six cauliflower seed lots, cultivars Sharon and Teresópolis Gigante). Piracicaba, USP-ESALQ, 2005.

\begin{tabular}{|c|c|c|c|c|c|c|}
\hline \multirow{3}{*}{ Lotes } & \multicolumn{6}{|c|}{ Cultivar Sharon } \\
\hline & \multirow{2}{*}{$\begin{array}{c}\text { VG }^{1} \\
\text { (índice) }\end{array}$} & $\mathbf{G}^{1}$ & $\mathrm{E}^{1}$ & EASS $^{1}$ & TA $^{1}$ & \multirow{2}{*}{$\frac{\mathrm{CE}^{1}}{\left(\mu \mathrm{S} \mathrm{cm} \cdot \mathrm{g}^{-1}\right)}$} \\
\hline & & \multicolumn{4}{|c|}{ (\%) } & \\
\hline 1 & $16,1 \mathrm{~A}$ & $98 \mathrm{~A}$ & $96 \mathrm{~B}$ & $87 \mathrm{~B}$ & 6,1 & $126,2 \mathrm{AB}$ \\
\hline 2 & $16,4 \mathrm{~A}$ & $99 \mathrm{~A}$ & $99 \mathrm{~A}$ & $98 \mathrm{~A}$ & 6,2 & $91,7 \mathrm{~A}$ \\
\hline 3 & $16,1 \mathrm{~A}$ & $98 \mathrm{~A}$ & $99 \mathrm{~A}$ & $98 \mathrm{~A}$ & 6,0 & 149,6 B \\
\hline \multirow[t]{2}{*}{$\mathrm{CV}(\%)$} & 1,6 & 1,4 & 1,4 & 3,6 & & 21,3 \\
\hline & \multicolumn{6}{|c|}{ Cultivar Teresópolis Gigante } \\
\hline \multirow[t]{2}{*}{ Lotes } & VG & $\mathbf{G}$ & $E$ & EASS & TA & $\mathrm{CE}$ \\
\hline & (índice) & \multicolumn{4}{|c|}{$(\%)$} & $\overline{\left(\mu \mathrm{S} \mathrm{cm} \cdot \mathbf{g}^{-1}\right)}$ \\
\hline 4 & $15,2 \mathrm{~A}$ & $94 \mathrm{~A}$ & $92 \mathrm{AB}$ & $88 \mathrm{~A}$ & 5,9 & $156,3 \mathrm{~A}$ \\
\hline 5 & $15,7 \mathrm{~A}$ & $96 \mathrm{~A}$ & $98 \mathrm{~A}$ & $93 \mathrm{~A}$ & 6,2 & $147,8 \mathrm{~A}$ \\
\hline 6 & $14,8 \mathrm{~A}$ & $91 \mathrm{~A}$ & $88 \mathrm{~B}$ & $92 \mathrm{~A}$ & 6,0 & 231,7 B \\
\hline CV(\%) & 4,30 & 4,60 & 5,1 & 4,5 & & 16,10 \\
\hline
\end{tabular}

Médias seguidas de mesma letra, dentro de cada cultivar, não diferem significativamente entre si, teste de Tukey, $\mathrm{p} \leq 0,05$ (means followed by the same letter, into each cultivar, did not differ from each other by Tukey's test, $\mathrm{p} \leq 0,05$ ); ${ }^{1} \mathrm{VG}=$ velocidade de germinação (speed of germination); $\mathrm{G}=$ germinação (germination); $\mathrm{E}=$ emergência de plântulas (seedling emergence); $\mathrm{EASS}=$ envelhecimento acelerado (accelerated aging); TA, = teor de água (moisture content); $\mathrm{CE}=$ condutividade elétrica (electrical conductivity).

e três lotes) para a cultivar Teresópolis Gigante. As médias foram comparadas pelo teste de Tukey $(\mathrm{p} \leq 0,05)$.

\section{RESULTADOS E DISCUSSÃO}

Para a cultivar Sharon, o lote 1 foi o de menor potencial fisiológico inicial, identificado pelos testes de emergência de plântulas e envelhecimento acelerado com uso de solução saturada de $\mathrm{NaCl}$ (Tabela 1). Porém, o teste de condutividade elétrica, identificou os lotes 2 e 3 como os de menor potencial fisiológico, embora apenas o último tenha diferido significativamente do lote 1 (Tabela 1). O teor de água inicial das sementes variou 0,2 pontos percentuais $(6,0$ a $6,2 \%)$, situando-se dentro do limite tolerável de 2 a 3 pontos percentuais (Marcos Filho, 1999), o que confirma a consistência dos procedimentos utilizados para a condução dos testes. Para a cultivar Teresópolis Gigante, os lotes 5 e 6 foram identificados como os de maior e menor potencial fisiológico, respectivamente (Tabela 1). O teor de água inicial das sementes variou entre 5,9 e 6,2\%. Desta maneira, os resultados mostram que os lotes de sementes de ambas as cultivares apresentavam diferenças quanto ao potencial fisiológico inicial, o que é interessante, pois a utilização de lotes com diferenças no potencial fisiológico e pertencentes a cultivares distintas permite verificar o grau de consistência da aplicação dos métodos empregados para o condicionamento fisiológico das sementes, para a espécie em estudo.

Quanto à marcha de absorção de água pelas sementes, verificou-se que a protrusão da raiz primária ocorreu após 36 horas de embebição para a cultivar Teresópolis Gigante, quando as sementes atingiram teor de água de aproximadamente $43 \%$ para os três lotes estudados. Já para a cultivar Sharon, a emissão da raiz primária ocorreu quando as sementes atingiram em torno de $39 \%$ de água, também após 36 horas de embebição. Considerou-se que as sementes atingiram a fase III da curva de absorção de água quando pelo menos $1 \%$ da amostra apresentava emissão da raiz primária.

Durante a execução dos tratamentos, verificou-se que as sementes da cultivar Sharon submetidas ao hidrocondicionamento entre duas folhas de papel toalha não alcançaram teor de água superior a 32\%, devido à insuficiên- 
Tabela 2 - Testes de germinação (velocidade e porcentagem) e de emergência de plântulas (velocidade e porcentagem) de sementes de couve-flor, cultivar Sharon, submetidas a diferentes métodos de condicionamento fisiológico (Germination test (speed and percentage) and seedling emergence (speed and percentage) of cauliflower seeds, Sharon cultivar, submitted to different priming procedures. Piracicaba, USP - ESALQ, 2005

\begin{tabular}{|c|c|c|c|c|c|c|c|c|c|c|c|c|c|c|}
\hline \multirow{3}{*}{$\begin{array}{l}\text { Tratamentos } \\
\text { Testemunha }\end{array}$} & \multicolumn{8}{|c|}{ Velocidade de germinação (índice) } & \multicolumn{6}{|c|}{ Germinação (\%) } \\
\hline & \multicolumn{2}{|c|}{ Lote 1} & \multicolumn{2}{|c|}{ Lote 2} & \multicolumn{3}{|c|}{ Lote 3} & Médias & \multicolumn{2}{|c|}{ Lote 1} & \multirow{2}{*}{$\frac{\text { Lote } 2}{99}$} & \multirow{2}{*}{\multicolumn{2}{|c|}{$\frac{\text { Lote } 3}{97}$}} & \multirow{2}{*}{$\begin{array}{c}\text { Médias } \\
98\end{array}$} \\
\hline & $15,3 \mathrm{ABC}$ & a & $14,6 \mathrm{C}$ & & 14,50 & & $\mathrm{~b}$ & 14,8 & 9 & & & & & \\
\hline Hidroc. 2 folhas $/ 32 \%$ & $15,5 \mathrm{AB}$ & $\mathrm{b}$ & $16,4 \mathrm{~A}$ & & $16,1 \mathrm{~A}$ & & $a b$ & 16,0 & 9 & & 99 & 98 & & 98 \\
\hline Hidroc. 4 folhas $/ 32 \%$ & $15,2 \mathrm{ABC}$ & $\mathrm{b}$ & $16,3 \mathrm{~A}$ & & $15,2 \mathrm{~B}$ & & $\mathrm{~b}$ & 15,5 & 9 & & 100 & 96 & & 98 \\
\hline Hidroc.4 folhas/38\% & $15,8 \mathrm{~A}$ & a & $16,5 \mathrm{~A}$ & & $16,3 A$ & & a & 16,2 & 9 & & 99 & 99 & & 98 \\
\hline Hidroc. 6 folhas $/ 32 \%$ & $15,4 \mathrm{ABC}$ & a & $15,9 \mathrm{AB}$ & & $15,9 \mathrm{~A}$ & & a & 15,8 & 9 & & 100 & 99 & & 99 \\
\hline Hidroc. 6 folhas/38\% & $16,0 \mathrm{~A}$ & a & $16,5 \mathrm{~A}$ & & $15,9 \mathrm{~A}$ & & a & 16,2 & 9 & & 99 & 97 & & 98 \\
\hline Osmoc.-0,2 MPa /32\% & $11,7 \mathrm{E}$ & a & $12,1 \mathrm{D}$ & & $11,9 \mathrm{E}$ & & a & 11,9 & 9 & & 99 & 98 & & 98 \\
\hline Osmoc.-0,2 MPa/38\% & $14,5 \mathrm{C}$ & $\mathrm{b}$ & $15,7 \mathrm{AB}$ & & $15,4 \mathrm{~A}$ & $A B C$ & a & 15,2 & 9 & & 99 & 99 & & 98 \\
\hline Osmoc.-0,1 MPa/32\% & $13,4 \mathrm{D}$ & $\mathrm{b}$ & $14,6 \mathrm{C}$ & & $13,9 \mathrm{D}$ & & $a b$ & 14,0 & 9 & & 100 & 98 & & 98 \\
\hline Osmoc.-0,1 MPa/38\% & $14,5 \mathrm{BC}$ & $\mathrm{a}$ & $15,2 \mathrm{BC}$ & & $14,7 \mathrm{C}$ & & $\mathrm{a}$ & 14,8 & 9 & & 99 & 99 & & 98 \\
\hline Médias & 14 & & & 5,4 & & 15,0 & & & 97 & & $99 a$ & 98 & & \\
\hline CV (\%) & & & & & 3,1 & & & & & & & 2,3 & & \\
\hline \multirow{2}{*}{ Tratamentos } & \multicolumn{7}{|c|}{ Velocidade de emergência (índice) } & \multicolumn{7}{|c|}{ Emergência (\%) } \\
\hline & \multicolumn{2}{|c|}{ Lote1 } & \multicolumn{2}{|c|}{ Lote 2} & \multicolumn{2}{|c|}{ Lote 3} & Médias & \multicolumn{2}{|c|}{ Lote 1} & \multicolumn{2}{|c|}{ Lote 2} & \multicolumn{2}{|c|}{ Lote 3} & Médias \\
\hline Testemunha & $8,9 \mathrm{BC}$ & $a b$ & $10,1 \mathrm{AB}$ & a & $7,8 \mathrm{CD}$ & $\mathrm{b}$ & 8,9 & $89 \mathrm{~B}$ & b & $97 \mathrm{AB}$ & a & $86 \mathrm{~B}$ & $\mathrm{~b}$ & 91 \\
\hline Hidroc. 2 folhas $/ 32 \%$ & $9,8 \mathrm{AB}$ & a & $10,9 \mathrm{AB}$ & a & $10,6 \mathrm{AB}$ & a & 10,4 & $92 \mathrm{AB}$ & $a b$ & $89 \mathrm{~B}$ & $\mathrm{~b}$ & $97 \mathrm{~A}$ & a & 93 \\
\hline Hidroc.4 folhas $/ 32 \%$ & $10,0 \mathrm{AB}$ & a & $10,6 \mathrm{AB}$ & a & $10,0 \mathrm{AB}$ & a & 10,2 & $97 \mathrm{AB}$ & a & $93 \mathrm{AB}$ & a & $98 \mathrm{~A}$ & a & 96 \\
\hline Hidroc.4 folhas/38\% & $11,2 \mathrm{~A}$ & a & $11,2 \mathrm{AB}$ & a & $11,6 \mathrm{~A}$ & a & 11,3 & $95 \mathrm{AB}$ & a & $99 \mathrm{~A}$ & a & $96 \mathrm{~A}$ & a & 97 \\
\hline Hidroc. 6 folhas $/ 32 \%$ & $8,1 \mathrm{CD}$ & $\mathrm{b}$ & $10,7 \mathrm{AB}$ & a & $10,5 \mathrm{AB}$ & $a$ & 9,8 & $94 \mathrm{AB}$ & $a b$ & $100 \mathrm{~A}$ & a & $92 \mathrm{AB}$ & $b$ & 95 \\
\hline Hidroc. 6 folhas $/ 38 \%$ & $10,9 \mathrm{~A}$ & a & $11,0 \mathrm{AB}$ & a & $11,0 \mathrm{~A}$ & $a$ & 11,0 & $97 \mathrm{AB}$ & a & $99 \mathrm{~A}$ & a & $94 \mathrm{AB}$ & a & 97 \\
\hline Osmoc.-0,2 MPa /32\% & $11,2 \mathrm{~A}$ & a & $11,6 \mathrm{~A}$ & a & $11,6 \mathrm{~A}$ & a & 11,5 & $95 \mathrm{AB}$ & a & $98 \mathrm{AB}$ & a & $95 \mathrm{~A}$ & a & 96 \\
\hline Osmoc.-0,2 MPa/38\% & $6,6 \mathrm{DE}$ & $a b$ & $5,8 \mathrm{C}$ & $\mathrm{b}$ & $7,3 \mathrm{D}$ & a & 6,6 & $96 \mathrm{AB}$ & a & $96 \mathrm{AB}$ & a & $96 \mathrm{~A}$ & a & 96 \\
\hline Osmoc.-0,1 MPa/32\% & $4,9 \mathrm{E}$ & $\mathrm{b}$ & $4,8 \mathrm{C}$ & $\mathrm{b}$ & $6,5 \mathrm{D}$ & a & 5,4 & $96 \mathrm{AB}$ & a & $99 \mathrm{~A}$ & a & $97 \mathrm{~A}$ & a & 97 \\
\hline Osmoc.-0,1 MPa/38\% & $10,5 \mathrm{AB}$ & $\mathrm{a}$ & $9,9 \mathrm{~B}$ & $\mathrm{ab}$ & $9,1 \mathrm{BC}$ & $\mathrm{b}$ & 9,8 & $98 \mathrm{~A}$ & a & $99 \mathrm{~A}$ & a & $94 \mathrm{AB}$ & a & 97 \\
\hline CV $(\%)$ & & & & 6,0 & & & & & & & 5,1 & & & \\
\hline
\end{tabular}

Médias seguidas de mesma letra maiúscula na coluna não diferem significativamente entre si, teste de Tukey, p d" 0,05 . Médias seguidas de mesma letra minúscula nas linhas, dentro de cada característica, não diferem significativamente entre si, teste de Tukey, p d" 0,05 (Means followed the same capital letter in the column did not differ from each other by Tukey's test, p d" 0,05. Means followed the same small letter in the row, into each treatment, did not differ from each other by Tukey's test, $p \leq 0,05$ ).

cia da quantidade de água disponível; assim, o hidrocondicionamento entre 2 folhas de papel até atingir teor de água de $38 \%$ não foi considerado para a análise estatística. Os teores de água planejados não foram atingidos porque a quantidade de água retida em duas folhas de papel toalha é insuficiente para que as sementes de couve-flor atinjam o grau de umidade mínimo necessário para a protrusão da raiz primária.

Verificou-se interação significativa para tratamentos e lotes sobre a velocidade de germinação e sobre a emergência de plântulas (velocidade e porcentagem). Apenas o efeito de lotes foi significativo sobre a porcentagem de germinação, destacando o lote 2 como o de maior potencial fisiológico (Tabela 2), confirmando a avaliação inicial dos lotes (Tabela 1). Sementes dos lotes 2 e 3 da cultivar Sharon submetidas ao hidrocondicionamento e ao osmocondicionamento a $-0,2 \mathrm{MPa} / 38 \%$ de água apresentaram velocidade de germinação superior à da testemunha (Tabela 2). Para o lote 1, o osmocondicionamento foi menos eficiente que o hidrocondicionamento, resultando em redução da velocidade de germinação em relação à testemunha, quando o teor de água foi ajustado para $32 \%$. De maneira geral, sementes submetidas ao hidrocondicionamento apresentaram velocidade de germinação superior à das osmocondicionadas. Em outros trabalhos, hidrocondicionamento foi considerado mais eficiente que o osmocondicionamento, favorecendo a velocidade de germinação de sementes de cebola (Caseiro et al., 2004) e de cou- ve-flor, principalmente quando a germinação foi avaliada sob baixa temperatura (Fujikura et al.,1993).

Para a cultivar Sharon, o potencial fisiológico inicial dos lotes não influenciou os efeitos do condicionamento fisiológico das sementes, avaliados pela velocidade de germinação. Houve benefícios à velocidade de germinação para o lote de maior potencial fisiológico (lote 2) e para o lote 3 , de potencial fisiológico inferior ao lote 2 (Tabela 2). Estes resultados são semelhantes aos obtidos por Thornton et al. (1993) e por Thornton \& Powell (1995) com sementes de couve-flor.

Não houve efeito do condicionamento fisiológico sobre a porcentagem de plântulas normais obtidas no teste de germinação para a cultivar Sharon (Tabela 2). Esse fato é esperado, uma vez que o 
Tabela 3. Testes de germinação e emergência de plântulas (velocidade e porcentagem) de três lotes de sementes de couve-flor da cultivar Teresópolis Gigante submetidos a diferentes métodos de condicionamento fisiológico (germination test and seedling emergence (speed and percentage) of cauliflower seeds, cultivar Teresópolis Gigante, submitted to different priming procedures). Piracicaba, USP-ESALQ, 2005.

\begin{tabular}{|c|c|c|c|c|c|c|c|c|c|c|c|}
\hline \multirow{2}{*}{ Tratamentos } & \multicolumn{5}{|c|}{ Velocidade de germinação (índice) } & \multicolumn{6}{|c|}{ Germinação (\%) } \\
\hline & \multirow{2}{*}{$\begin{array}{r}\text { Lote } 4 \\
15,6\end{array}$} & \multicolumn{2}{|c|}{ Lote 5} & Lote 6 & Médias & \multicolumn{2}{|c|}{ Lote 4} & Lote 5 & \multicolumn{2}{|c|}{ Lote 6} & Médias \\
\hline Testemunha & & \multicolumn{2}{|c|}{17,6} & 15,9 & $16,4 \mathrm{CD}$ & \multicolumn{2}{|c|}{89} & 96 & \multicolumn{2}{|c|}{93} & 93 \\
\hline Hidroc. 4 folhas $/ 34 \%$ & 15,6 & \multicolumn{2}{|c|}{16,5} & 15,2 & $15,7 \mathrm{D}$ & \multicolumn{2}{|c|}{95} & 97 & \multicolumn{2}{|c|}{92} & 95 \\
\hline Hidroc.4 folhas $/ 41 \%$ & 21,2 & \multicolumn{2}{|c|}{21,7} & 21,4 & $21,4 \mathrm{~A}$ & \multicolumn{2}{|c|}{95} & 95 & \multicolumn{2}{|c|}{95} & 95 \\
\hline Hidroc. 6 folhas $/ 34 \%$ & 15,6 & \multicolumn{2}{|c|}{16,8} & 15,1 & $15,8 \mathrm{D}$ & \multicolumn{2}{|c|}{94} & 97 & \multicolumn{2}{|c|}{92} & 94 \\
\hline Hidroc. 6 folhas $/ 41 \%$ & 20,8 & \multicolumn{2}{|c|}{21,3} & 21,8 & $21,3 \mathrm{~A}$ & & & 97 & & & 96 \\
\hline Osmoc.-0,2 MPa /34\% & 15,1 & 15,8 & & 6 & $15,2 \mathrm{D}$ & & & 99 & & & 96 \\
\hline Osmoc.-0,2 MPa/41\% & 21,7 & 22,8 & & ,2 & $21,9 \mathrm{~A}$ & & & 94 & & & 94 \\
\hline Osmoc.-0,1 MPa/34\% & 17,0 & 19,6 & & 0 & $17,9 \mathrm{BC}$ & & & 97 & & & 94 \\
\hline Osmoc.-0,1 MPa/41\% & 18,9 & 19,6 & &, 8 & 19,4 B & & & 97 & & & 96 \\
\hline Médias & $17,9 \mathrm{~b}$ & 19,1 & & $0 \mathrm{~b}$ & & & & $99 \mathrm{a}$ & & & \\
\hline $\mathrm{CV}(\%)$ & & & 6,0 & & & & & & 6,7 & & \\
\hline Tratamentoc & Veloci & dade de er & nergência & índice) & & & & ergênci & ia (\%) & & \\
\hline iralamemios & Lote 4 & Lote 5 & Lote 6 & Médias & & & Lo & e 5 & & & Médias \\
\hline Testemunha & 09,9 & 11,2 & 07,3 & $09,5 \mathrm{~B}$ & $94 \mathrm{~A}$ & $a$ & $97 \mathrm{AB}$ & $\mathrm{a}$ & $93 \mathrm{~A}$ & $\mathrm{a}$ & 95 \\
\hline Hidroc. 4 folhas $/ 34 \%$ & 10,1 & 09,6 & 10,4 & $10,0 \mathrm{~B}$ & $91 \mathrm{AB}$ & $b$ & $98 \mathrm{~A}$ & a & $90 \mathrm{AB}$ & $\mathrm{b}$ & 93 \\
\hline Hidroc.4 folhas/41\% & 12,8 & 11,8 & 10,4 & $11,7 \mathrm{AB}$ & $94 \mathrm{~A}$ & a & $95 \mathrm{ABC}$ & a & $95 \mathrm{~A}$ & a & 95 \\
\hline Hidroc. 6 folhas $/ 34 \%$ & 09,4 & 11,4 & 09,8 & $10,2 \mathrm{~B}$ & $90 \mathrm{AB}$ & $b$ & $96 \mathrm{ABC}$ & a & $88 \mathrm{AB}$ & b & 91 \\
\hline Hidroc. 6 folhas $/ 41 \%$ & 13,4 & 13,7 & 13,4 & $13,5 \mathrm{~A}$ & $95 \mathrm{~A}$ & a & $96 \mathrm{ABC}$ & a & $95 \mathrm{~A}$ & a & 95 \\
\hline Osmoc.-0,2 MPa /34\% & 09,7 & 10,1 & 09,9 & 09,9 B & $92 \mathrm{AB}$ & a & $92 \mathrm{ABC}$ & a & $91 \mathrm{AB}$ & a & 92 \\
\hline Osmoc.-0,2 MPa/41\% & 11,4 & 11,9 & 11,7 & $11,7 \mathrm{AB}$ & $94 \mathrm{~A}$ & a & $96 \mathrm{ABC}$ & a & $92 \mathrm{~A}$ & a & 94 \\
\hline Osmoc.-0,1 MPa/34\% & 09,7 & 10,5 & 10,0 & $10,0 \mathrm{~B}$ & $86 \mathrm{~B}$ & a & $89 \mathrm{C}$ & a & $84 \mathrm{~B}$ & a & 86 \\
\hline Osmoc.-0,1 MPa/41\% & 09,0 & 12,0 & 11,7 & $10,9 \mathrm{AB}$ & $76 \mathrm{C}$ & $b$ & $90 \mathrm{BC}$ & $a$ & $88 \mathrm{AB}$ & $\mathrm{a}$ & 85 \\
\hline $\mathrm{CV}(\%)$ & & & 4 & & & & & 3,7 & & & \\
\hline
\end{tabular}

Médias seguidas de mesma letra maiúscula na coluna não diferem significativamente entre si, teste de Tukey, $\mathrm{p} \leq 0,05$; médias seguidas de mesma letra minúscula nas linhas, dentro de cada característica, não diferem significativamente entre si, teste de Tukey, $\mathrm{p} \leq 0,05$ (means followed by the same capital letter in the column did not differ from each other by Tukey's test, $\mathrm{p} \leq 0,05$; Means followed by the same small letter in the row, into each treatment, did not differ from each other by Tukey's test, $\mathrm{p} \leq 0,05)$.

teste de germinação é conduzido sob condições ideais e o condicionamento fisiológico somente tem afetado a porcentagem de germinação quando as condições edafo-climáticas são adversas (Rao et al., 1987; Lima \& Nascimento, 2002; Lima, 2008). Por outro lado, verificou-se desempenho superior do lote 2 em relação aos lotes 1 e 3 (Tabela 2). Essa superioridade do lote 2 já havia sido detectada pelo teste de condutividade elétrica na avaliação inicial do potencial fisiológico dos lotes (Tabela 1).

De modo geral, o condicionamento fisiológico favoreceu a velocidade e porcentagem de emergência das plântulas, em relação à testemunha, nos lotes 1 e 3 (menor potencial fisiológico), não sendo eficiente para o lote 2 (maior potencial fisiológico), confirmando as observações de Nascimento (1998).

De maneira geral, o lote de maior potencial fisológico da cultivar Sharon (lote
2) foi favorecido pelo condicionamento fisiológico somente quanto à velocidade de germinação, enquanto os lotes de menor potencial fisológico foram favorecidos quanto à velocidade de germinação e à emergência de plântulas (velocidade e porcentagem). Portanto, os lotes de menor potencial fisiológico foram mais sensíveis ao tratamento condicionador. Resultados similares foram obtidos com sementes de couve-flor (Thornton \& Powell, 1995; Powell et al., 2000), cebola (Caseiro et al., 2004), melão (Nascimento \& Aragão, 2004) e aspargo (Bittencourt et al., 2005).

Para a cultivar Teresópolis Gigante, também não foi possível a utilização do hidrocondicionamento entre duas folhas de papel toalha, pois as sementes alcançaram o teor de água de $29 \%$, inferior aos planejados (34 e $41 \%$ ). Este fato deve ter ocorrido pela mesma razão comentada anteriormente para as semen- tes da cv. Sharon, embora a captação de água possa ser afetada pelo genótipo e condição fisiológica das sementes.

Observou-se que para a cultivar Teresópolis Gigante houve efeitos significativos da interação lotes e tratamentos sobre a emergência de plântulas (Tabela 3). O efeito de lotes foi significativo sobre a porcentagem de germinação, para todos os tratamentos. Verificou-se, ainda, efeito significativo de tratamentos sobre a velocidade de emergência de plântulas e de lotes e de tratamentos sobre a velocidade de germinação (Tabela 3).

As sementes hidrocondicionadas entre quatro e seis folhas de papel toalha e as osmocondicionadas a - $0,2 \mathrm{MPa}$, nos teores de água mais elevados, apresentaram maior velocidade de germinação que os demais tratamentos, incluindo a testemunha, considerando a $\mathrm{cv}$. Teresópolis Gigante (Tabela 3). O 
hidrocondicionamento das sementes entre seis folhas (duas camadas de três folhas) até alcançar teor de água de $41 \%$ promoveu efeitos benéficos sobre a velocidade de emergência de plântulas. No entanto, o mesmo não ocorreu quanto à porcentagem de emergência de plântulas (Tabela 3).

Para a cultivar Teresópolis Gigante, de modo geral, o hidrocondicionamento favoreceu o desempenho das sementes, quando o teor de água atingiu $41 \%$ (mais elevado). Essa mesma observação pode ser dirigida para as sementes da cultivar Sharon, indicando que a eficiência do condicionamento sobre o potencial fisiológico das sementes é mais evidente quando o período de embebição é prolongado. Nesta pesquisa, os menores teores de água (32 e 34\% respectivamente para as cultivares Sharon e Teresópolis Gigante) foram atingidos em aproximadamente 12 horas, enquanto os mais elevados (38 e $41 \%$ respectivamente para as cultivares Sharon e Teresópolis Gigante), em aproximadamente 36 horas. Provavelmente, os menores teores de água não foram suficientes para permitir a reativação do metabolismo para a germinação, devido à rápida absorção de água.

Os efeitos do condicionamento fisiológico sobre a velocidade de germinação e de emergência das plântulas não foram dependentes do potencial fisiológico inicial dos lotes da cultivar Teresópolis Gigante, diferindo das observações efetuadas para a cultivar Sharon. Provavelmente, isto tenha ocorrido devido à influência do genótipo ou a variações no histórico dos lotes de cada cultivar. A análise dos resultados permite afirmar que o efeito do genótipo não deve ser desprezado, de modo que a definição de protocolo para uso comercial do condicionamento físiológico de sementes de couve-flor deve ser estabele- cido após avaliação mais ampla, envolvendo diferentes cultivares.

Tendo como base os resultados obtidos na presente pesquisa, concluiu-se que o hidrocondicionamento entre quatro e seis folhas de papel toalha, até a obtenção de teores de água próximos aos necessários para a protrusão da raiz primária, é benéfico à velocidade de germinação e de emergência de plântulas de couve-flor e que o potencial fisiológico inicial dos lotes pode influenciar a resposta ao condicionamento fisiológico, dependendo do cultivar ou histórico dos lotes.

\section{AGRADECIMENTOS}

À empresa Sakata Seed Sudamerica Ltda pela cessão das sementes. À FAPESP e ao CNPq, pela concessão das bolsas de estudo e suporte financeiro ao projeto.

\section{REFERÊNCIAS}

BITTENCOURT MLC; DIAS DCFS; DIAS LAS; ARAÚJO EF. 2005. Germination and vigor of primed asparagus seeds. Scientia Agricola 62: 319-324.

BRASIL. Ministério da Agricultura e Reforma Agrária. 1992. Regras para análise de sementes. Brasília: SNDA/DNDV/CLAV. $365 \mathrm{p}$.

CASEIRO R; BENNETT MA; MARCOS FILHO J. 2004. Comparison of three priming techniques for onion seed differing in initial seed quality. Seed Science and Technology 32: 365-375.

FUJIKURA Y; KRAAK HL; BASRA AS; KARSSEN CM. 1993. Hydropriming, a simple and inexpensive priming method. Seed Science and Technology 21: 639-642.

HAMPTON JG; TeKRONY DM. (Ed.). 1995. INTERNATIONAL SEED TESTING ASSOCIATION-ISTA. Handbook of vigour test methods. $3^{\text {rd }}$ ed. $117 \mathrm{p}$.

LIMA LB; NASCIMENTO WM. 2002. Condicionamento osmótico de sementes de berinjela, visando a germinação em condições de temperaturas baixas. Horticultura Brasileira 20. Suplemento 1 CD-ROM.
LIMA LB. 2008. Avaliação do potencial fisiológico e métodos de condicionamento, secagem e armazenamento de sementes de pepino. Piracicaba: USP-ESALQ. 93p (Tese doutorado).

MAGUIRE JD. 1962. Speed of germination aid in selection and evaluation for seedling vigour. Crop Science 2: 176-177.

MARCOS FILHO J. 1999. Teste de envelhecimento acelerado. In: KRZYZANOWSKI FC; VIEIRA RD; FRANÇA NETO JB (ed.). Vigor de sementes: conceitos e testes. Londrina: ABRATES. p.1-24.

MARCOS FILHO J. 2005. Fisiologia de sementes de plantas cultivadas. Piracicaba: FEALQ. 495p.

NASCIMENTO WM. 1998. Condicionamento osmótico de sementes de hortaliças: potencialidades e implicações. Horticultura Brasileira 16: 106-109.

NASCIMENTO WM; ARAGÃO FAS. 2004. Muskmelon seed priming in relation to seed vigor. Scientia Agricola 61: 114-117.

POWELL AA; YULE LJ; JING H-C; GROOT SPC; BINO RJ; PRITCHARD HW. 2000. The influence of aerated hydration seed treatment on seed longevity as assessed by the viability equations. Journal of Experimental Botany 51: 2031-2043.

RAO SC; AKERS SW; AHRING RM. 1987. Priming brassica seed to improve emergence under different temperatures and soil moisture conditions. Crop Science 27: 1050-1053.

SANTOS CMR; MENEZES NL. 2000. Tratamentos pré-germinativos em sementes de alface. Revista Brasileira de Sementes 22: 253-258.

THORNTON JM; COLLINS ARS; POWELL AA. 1993. The effect of aerated hydration on DNA synthesis in embryos of Brassica oleracea L. Seed Science Research 3: $195-$ 199.

THORNTON JM; POWELL AA. 1995. Prolonged aerated hydration for improvement of seed quality in Brassica oleracea L. Annals of Applied Biology 127: 183-189.

VILLELA FA; DONI FILHO L; SEQUEIRA EL. 1991. Tabela de potencial osmótico em função da concentração de polietilenoglicol 6.000 e da temperatura. Pesquisa Agropecuária Brasileira 26: 1957-1968. 